\title{
Lipoprotein(a) should be taken much more seriously
}

\author{
“...genetic studies have succeeded in demonstrating that lipoprotein(a) is not just a biomarker of increased risk \\ of IHD but a causal factor."
}

Lipoprotein(a) was first discovered in 1963 by Kaare Berg [1] and since the first study demonstrating an association with ischemic heart disease, it has been the subject of numerous epidemiologic, animal and in vitro studies [2]. Lipoprotein(a) consists of what is essentially a cholesterol-containing low-density lipoprotein particle covalently bound to a glycoprotein, Apo (a) [2]. Apo(a) is structurally very similar to plasminogen and has been shown to interfere with the activation and function of plasmin-inhibiting fibrinolysis. The structure of lipoprotein(a) suggests that it may be able to directly contribute to the development of atherosclerosis and thrombosis and, thus, to ischemic heart disease (IHD) and myocardial infarction (MI). Data from in vitro [3] as well as animal studies [4] support this hypothesis, and has implicated lipoprotein(a) in foam-cell formation, smooth muscle cell proliferation and plaque inflammation and instability [3-5]. Recently, lipoprotein(a) has also been shown to carry oxidized phospholipids [6] implicated in coronary artery disease [7]. Furthermore, the presence of lipoprotein(a) in atherosclerotic plaques but not in normal human arteries has been demonstrated $[3,4,8]$.

\section{Epidemiologic studies of the association of lipoprotein(a) with risk of ischemic heart disease}

Early cross-sectional studies very consistently demonstrated an association of elevated levels of lipoprotein(a) levels with risk of IHD [2]. However, initial results from subsequent prospective studies were inconsistent $[9,10]$, most likely owing to the use of long-term frozen samples and lipoprotein(a) assays subject to bias. At present, although, convincing evidence from later prospective studies and meta-analyses has accumulated over the years, demonstrating a clear association of elevated levels of lipoprotein(a) with increased risk of IHD and MI [11-13]. Evidence from recent prospective studies indicates that, in particular, extreme levels of lipoprotein(a) are associated with a markedly increased risk of IHD [14-16]. In the Copenhagen City Heart Study, a large prospective study of the general population, individuals with levels above the 90th or 95th percentiles of the lipoprotein(a) concentration distribution have a two- to three-fold increased risk of MI compared with individuals with low levels [16]. However, observational epidemiologic studies, no matter how well designed, cannot provide definite evidence of causal associations.

\section{Genetic epidemiologic studies \& causality}

Evidence of causality in the medical field is most convincingly provided by randomized, controlled trials. However, at present, no such trial of the effect of lowering levels of lipoprotein(a) on risk of IHD or MI has been conducted. The current lack of a specific and effective lipoprotein(a)lowering agent has probably impeded the conduct of any such trials.

\section{"The structure of lipoprotein(a) suggests that it may be able to directly contribute to the development of atherosclerosis and thrombosis..."}

Lacking evidence from randomized, controlled trials, genetic studies designed as so-called Mendelian randomization trials can be used to provide evidence of causality [17-19]. The term Mendelian randomization refers to the random assortment of genes from parents to offspring at the time of conception. In genetic-association studies, groups of individuals defined by their different genotypes are compared, and the laws of Mendelian genetics make such studies comparable to randomized clinical trials, where the randomization process probably leads to a similar distributions of known and unknown confounders in control and intervention groups. Thus, associations of genotype with risk of disease are, generally speaking, unconfounded and, therefore, may be

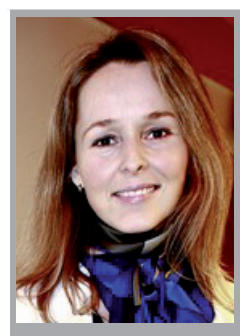

Pia R Kamstrup

Author for correspondence:

Department of Clinical

Biochemistry, Herlev Hospital,

Copenhagen University

Hospital, Herlev Ringvej 75,

DK-2730 Herlev, Denmark

Tel.: +45 4488 3842;

Fax: +454488 3311;

piroka01@heh.regionh.dk

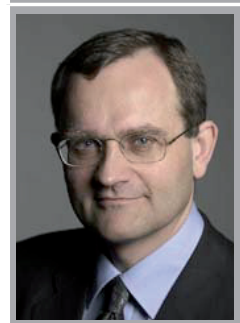

Børge G Nordestgaard

Department of Clinical

Biochemistry, Herlev Hospital

Copenhagen University

Hospital, Denmark

Tel.: +4544883297

Fax: +4544883311

brno@heh.regionh.dk

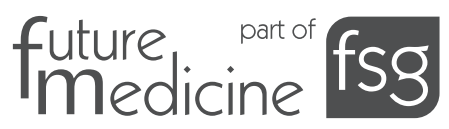


used to assess causality. Put simply, in a Mendelian randomization study, association of elevated levels of lipoprotein(a), as well as association of genetic variation raising levels of lipoprotein(a), with risk of IHD would suggest causality.

Lipoprotein(a) levels are predominantly genetically determined and not greatly influenced by lifestyle factors and may vary up to a 1000-fold between individuals [2]. The fact that levels are primarily genetically determined makes lipoprotein(a) an attractive target for a Mendelian randomization study. The LPA gene on chromosome 6 codes for the Apo(a) moiety of lipoprotein(a) [2]. Variation in this gene has a profound effect on plasma levels of lipoprotein(a). Of particular importance is the so-called kringle IV type 2 (KIV-2) size polymorphism or copy-number variant defined by a $5.6-\mathrm{kb}$ large sequence that may occur between two- and more than 40-times per allele, dependent on the specific genotype. This polymorphism determines the size of the expressed Apo(a) protein [2] and explains at least $25 \%$ of the total variation in plasma concentration of lipoprotein(a) in the general population [16]; this is a huge fraction explained by a single copy-number variant and makes this variant ideal for testing causality between lipoprotein(a) levels and risk of IHD and MI.

We recently demonstrated in three independent studies, including two general population studies (the Copenhagen City Heart Study and the Copenhagen General Population Study), and including a total of more than 40,000 individuals, that the lipoprotein(a) KIV-2 size polymorphism is unequivocally associated with risk of MI [16]. This observation is supported by data from former smaller case-control studies of the KIV-2 size polymorphism [20-22]. The previously demonstrated association of elevated levels of lipoprotein(a) with risk of MI, and the association of the KIV-2 size polymorphism with plasma levels of lipoprotein(a), were also confirmed in our studies [16]. Thus, according to the principles of Mendelian randomization studies, elevated levels of lipoprotein(a) are causally associated with risk of MI. This observation makes lipoprotein(a) a potential target for prophylactic interventions directed at lowering levels of lipoprotein(a) to reduce risk of MI.

\section{Future perspective}

Genetic studies of polymorphisms influencing levels of lipoprotein(a) and risk of IHD have provided a strong argument for a causal association of elevated levels of lipoprotein(a) with increased risk of IHD $[16,22,23]$. However, final proof of causality in the form of randomized, controlled trials demonstrating that lowering of lipoprotein(a) levels leads to decreased risk of IHD are presently lacking. High doses of nicotinic acid are known to reduce levels of lipoprotein(a) [24]. Unfortunately nicotinic acid at high doses is not well tolerated, and also may not be effective enough in lowering levels of lipoprotein(a). Furthermore, a randomized, controlled trial of the effect of lowering lipoprotein(a) levels by nicotinic acid on risk of IHD would also not be easily interpreted as nicotinic acid has pleiotropic effects on the lipid profile.

\section{"Despite numerous past studies there are still unknowns, such as the exact mechanism of how lipoprotein(a) causes MI..."}

In summary, genetic studies have succeeded in demonstrating that lipoprotein(a) is not just a biomarker of increased risk of IHD but a causal factor. In addition, the recent large meta-analysis of individual data from 126,634 participants from 36 prospective studies demonstrates the robustness of the association between elevated levels of lipoprotein(a) and increased risk of cardiovascular disease [13]. Together, this warrants that lipoprotein(a) should be taken much more seriously in the future, for cardiovascular risk examination and potentially as a target for drug therapy. Therefore, it is our hope that renewed interest in lipoprotein(a) may be spurred. Despite numerous past studies there are still unknowns, such as the exact mechanism of how lipoprotein(a) causes MI, is it primarily via increased atherosclerosis or increased thrombosis or a combination of both? Furthermore, the site and mechanism of lipoprotein(a) degradation and/or excretion in humans is not known. Finally, we need randomized, controlled trials to explore the benefits of lowering levels of lipoprotein(a) and, that again, requires the development of suitable lipoprotein(a)-lowering drugs.

Financial \& competing interests disclosure

Pia R Kamstrup reported being a consultant for Abbott. Børge $G$ Nordestgaard reported being a consultant for Abbott and AstraZeneca, and receiving lecture honoraria from Merck, AstraZeneca, Pfizer, Sanofi-Aventis and Boehringer-Ingelheim. The authors have no other relevant affliations or financial involvement with any organization or entity with a financial interest in or financial conflict with the subject matter or materials discussed in the manuscript apart from those disclosed.

No writing assistance was utilized in the production of this manuscript. 


\section{Bibliography}

1 Berg K: A new serum type system in man - the LP system. Acta Pathol. Microbiol. Scand. 59, 369-382 (1963).

2 Utermann G: Lipoprotein(a). In: The Metabolic and Molecular Bases of Inherited Disease (2001 Edition). Scriver CR, Beaudet AL, Sly WS, Valle D (Eds). McGraw-Hill, Medical Publishing Division, NY, USA, 2753-2787 (2006).

3 Deb A, Caplice NM: Lipoprotein(a): new insights into mechanisms of atherogenesis and thrombosis. Clin. Cardiol. 27(5), 258-264 (2004).

4 Boffa MB, Marcovina SM, Koschinsky ML: Lipoprotein(a) as a risk factor for atherosclerosis and thrombosis: mechanistic insights from animal models. Clin. Biochem. 37(5), 333-343 (2004).

5 Nielsen LB, Juul K, Nordestgaard BG: Increased degradation of lipoprotein(a) in atherosclerotic compared with nonlesioned aortic intima-inner media of rabbits: in vivo evidence that lipoprotein(a) may contribute to foam cell formation. Arterioscler. Thromb. Vasc. Biol. 18(4), 641-649 (1998).

6 Edelstein C, Pfaffinger D, Hinman J et al.: Lysine-phosphatidylcholine adducts in kringle V impart unique immunological and potential pro-inflammatory properties to human apolipoprotein(a). J. Biol. Chem. 278(52), 52841-52847 (2003).

7 Tsimikas S, Brilakis ES, Miller ER et al:: Oxidized phospholipids, Lp(a) lipoprotein, and coronary artery disease. N. Engl. J. Med. 353(1), 46-57 (2005).

8 Nielsen LB, Grønholdt ML, Schroeder TV, Stender S, Nordestgaard BG: In vivo transfer of lipoprotein(a) into human atherosclerotic carotid arterial intima. Arterioscler. Thromb. Vasc. Biol. 17(5), 905-911 (1997).
9 Alfthan G, Pekkanen J, Jauhiainen M et al.: Relation of serum homocysteine and lipoprotein(a) concentrations to atherosclerotic disease in a prospective Finnish population based study. Atherosclerosis 106(1), 9-19 (1994).

10 Ridker PM, Hennekens CH, Stampfer MJ: A prospective study of lipoprotein(a) and the risk of myocardial infarction. JAMA 270 (18), 2195-2199 (1993).

11 Craig WY, Neveux LM, Palomaki GE, Cleveland MM, Haddow JE: Lipoprotein(a) as a risk factor for ischemic heart disease: metaanalysis of prospective studies. Clin. Chem. 44(11), 2301-2306 (1998).

12 Danesh J, Collins R, Peto R: Lipoprotein(a) and coronary heart disease - meta-analysis of prospective studies. Circulation 102(10), 1082-1085 (2000).

13 Erqou S, Kaptoge S, Perry PL et al: : Lipoprotein(a) concentration and the risk of coronary heart disease, stroke, and nonvascular mortality. JAMA 302(4), 412-423 (2009).

14 Danik JS, Rifai N, Buring JE, Ridker PM: Lipoprotein(a), measured with an assay independent of apolipoprotein(a) isoform size, and risk of future cardiovascular events among initially healthy women. JAMA 296(11), 1363-1370 (2006).

15 Kamstrup PR, Benn M, Tybjærg-Hansen A, Nordestgaard BG: Extreme lipoprotein(a) levels and risk of myocardial infarction in the general population: the Copenhagen City Heart Study. Circulation 117(2), 176-184 (2008).

16 Kamstrup PR, Tybjærg-Hansen A, Steffensen R, Nordestgaard BG: Genetically elevated lipoprotein(a) and increased risk of myocardial infarction. JAMA 301(22), 2331-2339 (2009).
17 Clayton D, McKeigue PM: Epidemiological methods for studying genes and environmental factors in complex diseases. Lancet 358(9290), 1356-1360 (2001).

18 Smith GD, Ebrahim S: Mendelian randomization: prospects, potentials, and limitations. Int. J. Epidemiol. 33(1), 30-42 (2004).

19 Zacho J, Tybjærg-Hansen A, Jensen JS, Grande P, Sillesen H, Nordestgaard BG: Genetically elevated C-reactive protein and ischemic vascular disease. $N$. Engl. J. Med. 359(18), 1897-1908 (2008).

20 Holmer SR, Hengstenberg C, Kraft HG et al.: Association of polymorphisms of the apolipoprotein(a) gene with lipoprotein(a) levels and myocardial infarction. Circulation. 107(5), 696-701 (2003).

21 Sandholzer C, Saha N, Kark JD et al.: Apo(a) isoforms predict risk for coronary heart disease. A study in six populations. Arterioscler. Thromb. 12(10), 1214-1226 (1992).

22 Kraft HG, Lingenhel A, Kochl S et al: : Apolipoprotein(a) kringle IV repeat number predicts risk for coronary heart disease. Arterioscler. Thromb. Vasc. Biol. 16(6), 713-719 (1996).

23 Kamstrup PR, Tybjærg-Hansen A, Steffensen R, Nordestgaard BG: Pentanucleotide repeat polymorphism, lipoprotein(a) levels, and risk of ischemic heart disease. J. Clin. Endocrinol. Metab. 93(10), 3769-3776 (2008).

24 Carlson LA, Hamsten A, Asplund A: Pronounced lowering of serum levels of lipoprotein $\mathrm{Lp}(\mathrm{A})$ in hyperlipemic subjects treated with nicotinic-acid. J. Intern. Med. 226(4), 271-276 (1989). 